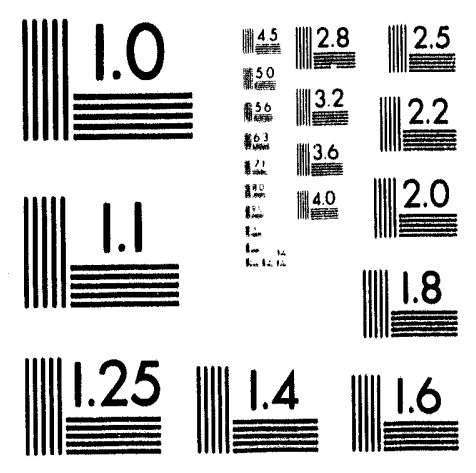



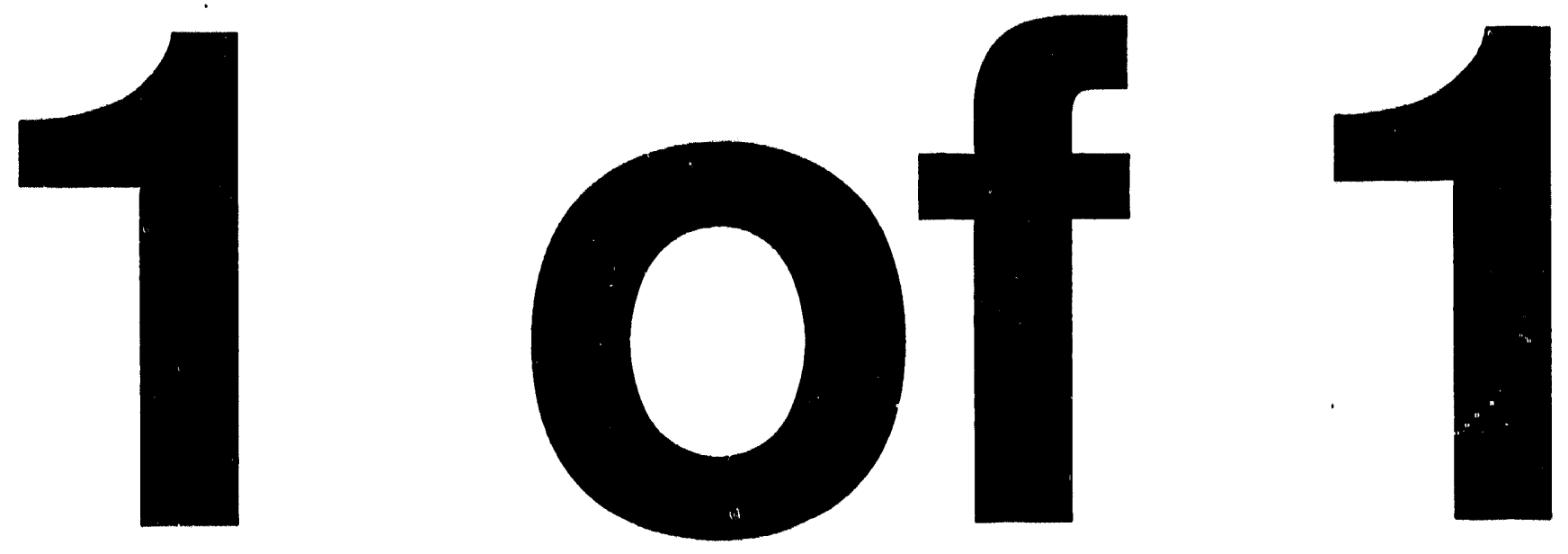
LA-UR- $93 \cdot 2895$

Title:

\title{
THE ACCELERATOR TRANSMUTATION OF WASTE CONCEPT OVERVIEW
}

\section{Author(s):}

\author{
Harry J. Dewey
}

\section{-CEIVED \\ OCT $0>1993$ \\ OSTI}

Submitted to:

For presentation at Annual ANS Meeting in San Diego held on June 19-25, 1993

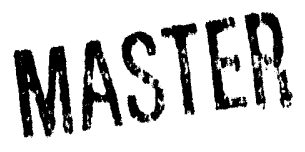

\section{Los Alamos}

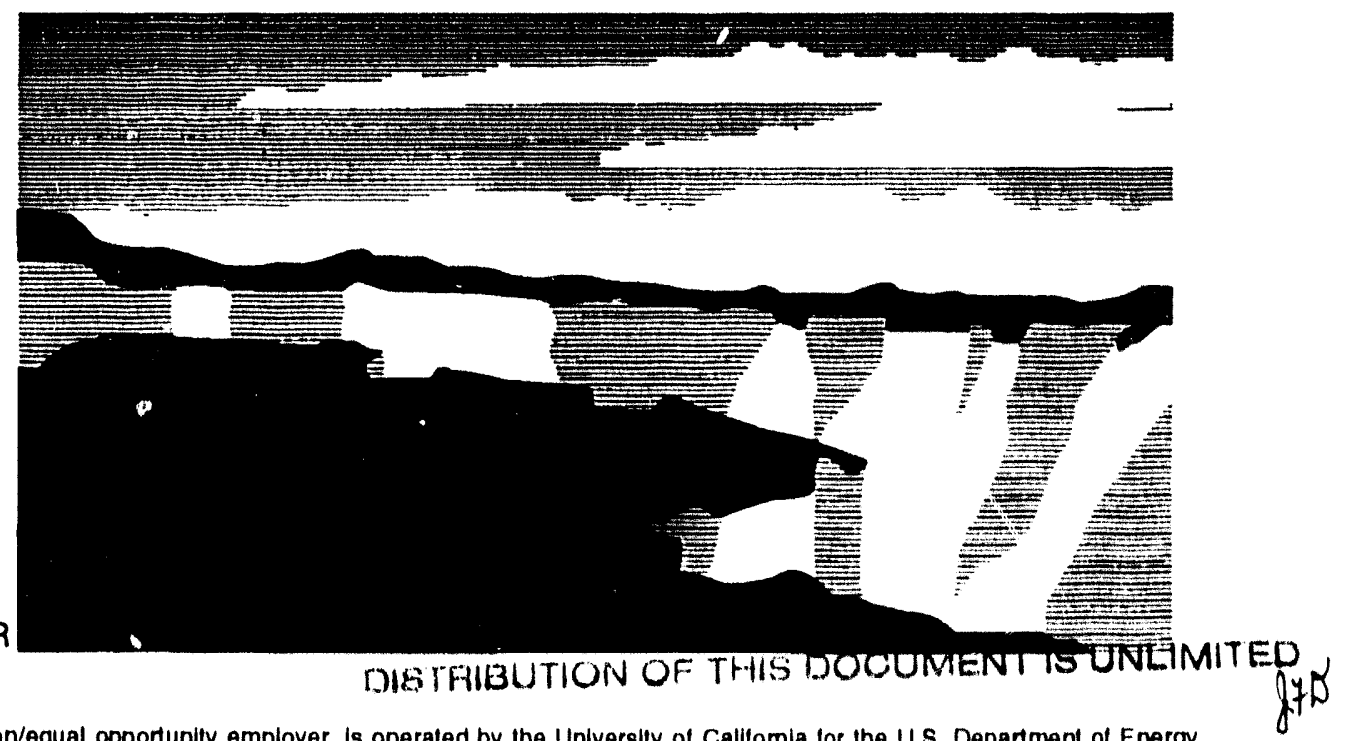

Los Alamos National Laboratory, an afflirmative action/equal opportunity employer, Is operated by the University of Calliomla lor the U.S. Department of Energy under contract W-7405-ENG-36. By acceplance of this anticle, the publisher recognizes that the U.S. Government retains a nonexciusive, royally. Iree license to publish or reproduce the published form of this contrlbution, or to altow olhers to do so, lor U.S. Government purposes. The Los Alamns Nalional Laboratory requesis that the putlisher Identlfy this article as work pertormed under the ausplces of the U.S. Department of Energy. 


\title{
THE ACCELERATOR TRANSMUTATION OF WASTE (ATW) CONCEPT OVERVIEW
}

\author{
Harry J. Dewey \\ Los Alamos National Laboratory \\ Los Alamos, New Mexico 87545 USA
}

The ATW concept is aimed at destroying key long-lived radionuclides (both actinides and fission products) in nuclear wastes, thereby reducing the long term risks associated with the storage of such wastes. This technology could evolve into an approach to the production of fission power, utilizing abundant natural fuels and producing minimal long-lived nuclear waste.

An ATW system would consist of the following components: (1) a proton accelerator, (2) a heavy metal target, (3) a moderating blanket, (4) a thermal to electric power conversion plant, and (5) a chemical separation facility. The linear accelerator provides a medium energy, high current proton beam that is directed at heavy metal target. The target converts the proton beam, through spallation reactions, into an intense neutron flux that is thermalized in the blanket region surrounding the arget. The radioactive material to be transmuted is circulated through the blanket where it undergoes neutron induced reactions. Long-lived fission products undergo $(n, \gamma)$ reactions followed by $\beta$ decay, producing short-lived or stable products. The actinides are fissioned, producing additional neutrons and an assortment of fission products. The fission energy from actinide burning is converted to electrical power and a portion is used to power the accelerator. The remainder can be sold to the commercial power grid. On-line chemical separations remove stable and short-lived fission products to reduce parasitic absorption in the blanket and to prevent further activation of these materials to long-lived radionuclides. Subsequent papers at this meeting will address the accelerator, target, and blanket technologies in greater detail.

The ATW approach to transmutation has a number of advantages over more conventional approaches that utilize nuclear reactors. One of the key advantages stems from the "extra" neutrons provided by the production target. Virtually any feed stream of longlived fission products and actinides can transmuted in such a system, ranging from a system that transmutes defense wastes containing fission products such as technetium and iodine to a system that transmutes a pure actinide feed such as weapons grade plutonium. The decision regarding which long-lived fission products to transmute and which to store can be based on an analysis of costs and benefits, not being limited by the number of neutrons produced in a fission event. Ultimately, it may be possible to destroy the long-lived species completely enough so that all wastes can be safely stored on site.

The neutrons from the target also allow the blanket to operate in a subcritical mode. This can provide significant safety advantages, depending on how far from criticality the system is designed to operate.

The combination of high neutron flux with a nearly thermal spectrum allows low inventories of waste in the blanket region for a given transmutation rate. Such low inventories reduce the risk for many accident scenarios, and also reduce the end-of-life residues. When coupled with high burn rates, low inventories have significant impact on time scales required to achieve overall fuel cycle inventory reduction.' 
The chemical separations required for ATW represent a demanding, but workable, chnllenge. The total material inventory for the system includes not only the material in the blanket but also material in the flow loop outside the blanket, material being cooled prior to processing, and material being processed. Thus, to minimize overall inventories, cooling times should be of the same order of magnitude as the residence times in the blanket flow loop. Under these conditions, chemical processes should be reasonably robust with respect to radiolytic decomposition. We have explored cooling times as short as five days and have found chemical separations that can be accomplished under such conditions. Waste stream minimization is also a key requirement in order to produce a favorable material balance for the system. Our beginning selection of chemical processes emphasize material recycle.

The most efficient extraction of power from the system can be accomplished by operating at high temperatures. The use of molten salt as the carrier medium can allow operation at both high temperature and near ambient pressure. Experience gained on the molten salt reactor program at Oak Ridge National Laboratory in the 1960's and 70's may be quite useful in developing a molten salt ATW system. However, the detailed separations required for such an ATW system will require a considerable development effort. We have also been exploring the use of physical separations to separate actinides and their fission products from each other and from the molten salt solvent. One example of such physical separations is the use of liquid centrifugation. Another approach could involve fractional crystallization.

Chemical separations in aqueous media are much better developed and we have proposed a separation scheme based a $\mathrm{D}_{2} \mathrm{O}$ carrier system that serves as an illustrative example, allowing us to perform beginning material balance calculations. The results of these studies are encouraging. Required separation factors are attainable and most, if not all, of the waste streams from chemical processing will meet low level waste disposal requirements. In addition, the quantities of waste generated from radiolytic decomposition appear to be manageable. Such a system is not optimized. Even so, such systems produce an attractive net production of electricity under conditions of significant waste reduction and favorable material balance.

1. T. H. Pigford and J. S. Choi, "Inventory Reduction Factors for Actinide-Burning Liquid-Metal Reactors," Trans. Am. Nucl. Soc., 64, 123-5 (1991). 

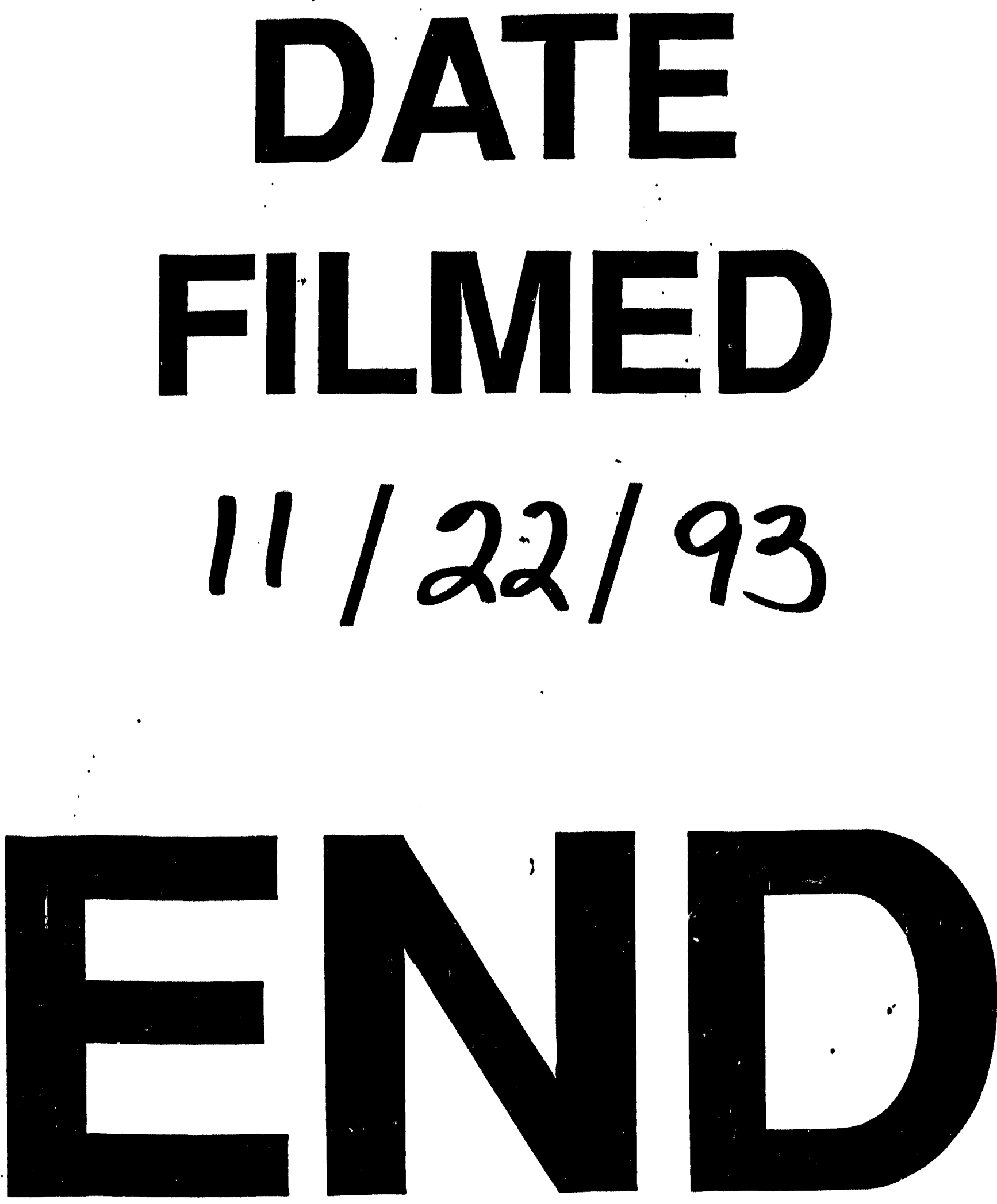

-

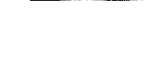

$$
\longrightarrow
$$

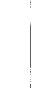

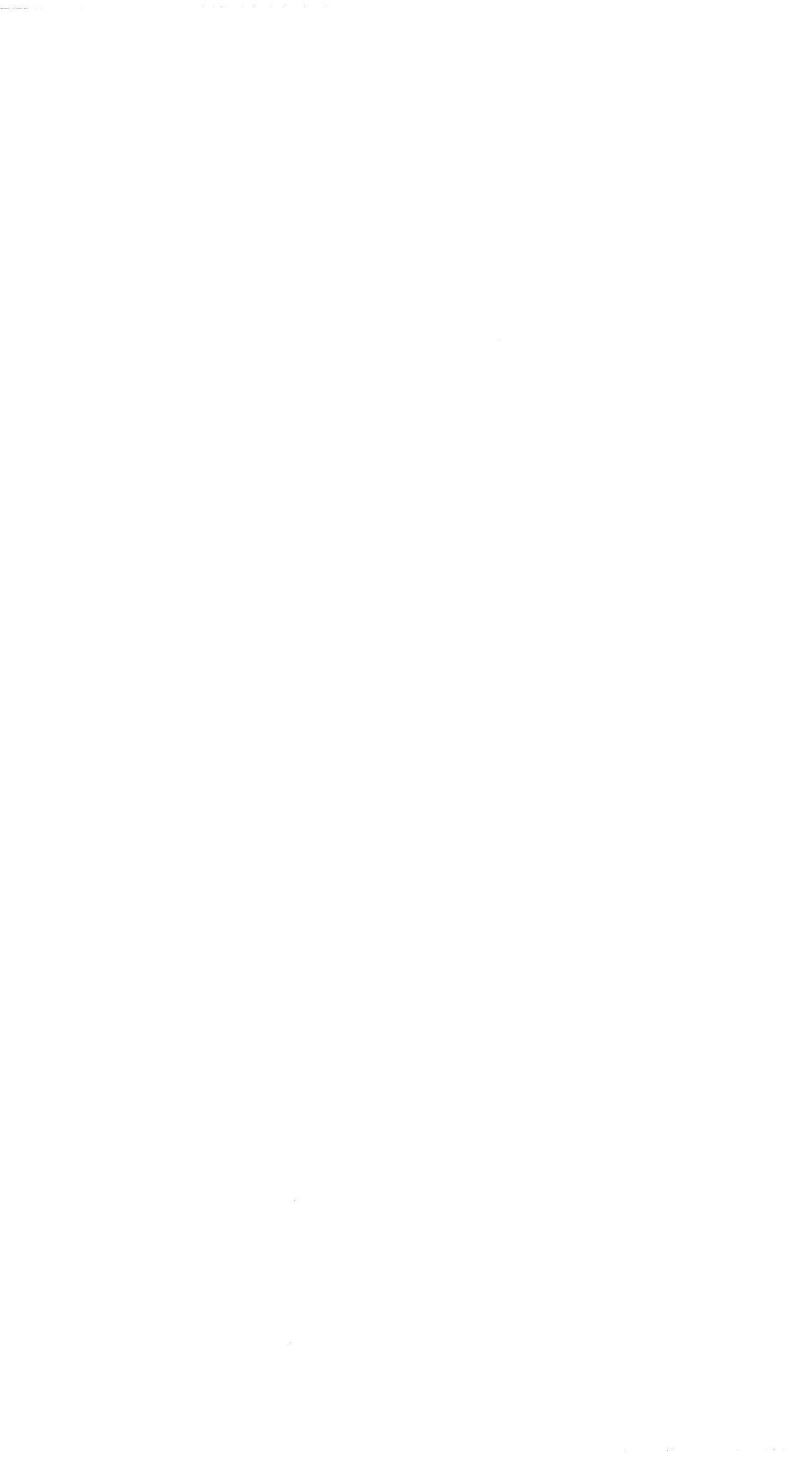

